\title{
Spin waves throughout the Brillouin zone and magnetic exchange coupling in ferromagnetic metallic manganites $\mathbf{L a}_{1-x} \mathbf{C a}_{x} \mathbf{M n O}_{3}(x=0.25,0.30)$
}

\author{
F. Ye, ${ }^{1, \text { f }}$ Pengcheng Dai, ${ }^{2,1}$ J. A. Fernandez-Baca, ${ }^{1,2}$ D. T. Adroja,${ }^{3}$ T. G. Perring, ${ }^{3}$ Y. Tomioka, ${ }^{4}$ and Y. Tokura ${ }^{4,5}$ \\ ${ }^{1}$ Neutron Scattering Science Division, Oak Ridge National Laboratory, Oak Ridge, Tennessee 37831-6393, USA \\ ${ }^{2}$ Department of Physics and Astronomy, The University of Tennessee, Knoxville, Tennessee 37996-1200, USA \\ ${ }^{3}$ ISIS facility, Rutherford Appleton Laboratory, Chilton, Didcot OX11 0QX, UK \\ ${ }^{4}$ Correlated Electron Research Center (CERC), Tsukuba 305-0046, Japan \\ ${ }^{5}$ Department of Applied Physics, University of Tokyo, Tokyo 113-8656, Japan
}

(Dated: October 25, 2018)

\begin{abstract}
Using time-of-flight and triple-axis inelastic neutron spectroscopy, we determine spin wave excitations throughout the Brillouin zone for ferromagnetic manganites $\mathrm{La}_{1-x} \mathrm{Ca}_{x} \mathrm{MnO}_{3}(x=0.25,0.3)$ in their low temperature metallic states. While spin wave excitations in the long wavelength limit (spin stiffness $D$ ) have similar values for both compounds, the excitations near the Brillouin zone boundary of $\mathrm{La}_{0.7} \mathrm{Ca}_{0.3} \mathrm{MnO}_{3}$ are considerable softened in all symmetry directions compared to that of $\mathrm{La}_{0.75} \mathrm{Ca}_{0.25} \mathrm{MnO}_{3}$. A Heisenberg model with the nearest neighbor and the fourth neighbor exchange interactions can describe the overall dispersion curves fairly well. We compare the data with various theoretical models describing the spin excitations of ferromagnetic manganites.
\end{abstract}

PACS numbers: 75.30.Ds, 61.12.-q, 71.30.+h, 72.15.Gd

\section{INTRODUCTION}

The experimental investigation of spin dynamical properties in doped manganese perovskite $A_{1-x} B_{x} \mathrm{MnO}_{3}$, where $A$ is the trivalent ion $\left(\mathrm{La}^{3+}, \mathrm{Pr}^{3+}, \mathrm{Nd}^{3+}\right.$, etc) and $B$ is the divalent ion $\left(\mathrm{Ca}^{2+}\right.$ or $\left.\mathrm{Sr}^{2+}\right)$, is essential to the understanding of spin-spin interactions in these materials. At hole-doping level $x \approx 0.30$, these so-called colossal magnetoresistance (CMR) compounds exhibit an unusually large change in electrical resistance in response to a magnetic field and changes from a paramagnetic to a ferromagnetic state $\stackrel{1,2.3}{=}$ The $\mathrm{Mn} 3 d$ levels in the $\mathrm{Mn}^{3+} / \mathrm{Mn}^{4+}$ mixed valent system, split by the oxygen octahedral crystal field to a lower energy $t_{2 g}$ triplet and a higher energy $e_{g}$ doublet, are filled according to the Hund's rule such that all spins are aligned on a given site by a large intra-atomic exchange coupling $J_{H}$. The basic microscopic mechanism responsible for the CMR effect is the double-exchange (DE) interaction, $\underline{\underline{4}}$ where ferromagnetism and electrical conductivity arise from hopping of the itinerant $e_{g}$ electrons with kinetic energy $t$ from trivalent $\mathrm{Mn}^{3+}$ to tetravalent $\mathrm{Mn}^{4+}$ sites. In its simplest form, the Hamiltonian of a DE model can be described as a single band of itinerant $e_{g}$ electrons interacting with localized core spins by the Hund's rule exchange $J_{H}$. Since $J_{H}$ is much larger than $t$, the kinetic energy of itinerant $e_{g}$ electrons is minimal when all electron spins are parallel, i.e., the ground state is a metallic ferromagnet.

Although DE interaction is believed to be responsible for the ferromagnetism and electron conductivity in CMR compounds, whether the magnetic excitations of such a model can be discussed in terms of an equivalent ferromagnetic Heisenberg model is still not clear. In the strong coupling limit $\left(t / J_{H} \rightarrow 0\right)$, Furukawa ${ }^{5}$ has shown that the DE model can be mapped onto the Heisenberg
Hamiltonian with only the nearest neighbor (NN) exchange coupling. In this scenario, the magnitude of the exchange coupling $J$ associated with ferromagnetic spin waves should scale with the Curie temperature $T_{c}$, kinetic energy $t$ and doping $x \stackrel{6,7,8,9}{ }$ Experimentally, the initial measurements on $\mathrm{La}_{0.7} \mathrm{~Pb}_{0.3} \mathrm{MnO}_{3}$ suggest that a simple NN Heisenberg model is sufficient to account for the entire spin wave dispersion relation ${ }^{\underline{7}}$ and the exchange coupling obtained from such model also yields, to within $15 \%$, the correct $T_{c}$ of the compound. However, later experimental measurements indicate that spin wave excitations of most $A_{1-x} B_{x} \mathrm{MnO}_{3}$ manganites with $x \approx 0.3$ renormalize near the zone boundary (ZB) with large softening and damping, $10,11,12,13,14$ Furthermore, the spin wave stiffness constant $D$ and the NN magnetic exchange coupling $J$ are weakly dependent on $T_{c}$ and doping level $x$ in metallic ferromagnetic (FM) manganites $\frac{15,16,17}{17}$

It is now well established that a Heisenberg model with $\mathrm{NN}$ exchange coupling is insufficient to describe the dispersion relation of CMR manganites, several possible microscopic mechanisms have been proposed to address the unusual features of spin wave excitations near the ZB. First, realistic calculations based on the DE mechanism with consideration of the finite kinetic energy $t$ and the effect of on-site Coulomb repulsion show that spin waves in the DE model do not map to the Heisenberg Hamiltonian with simple NN exchange coupling. $\underline{18}$ This model, however, predicts a doping dependence on $D$ which is not observed experimentally. Second, the observed ZB spin wave softening may be due to the conduction electron band filling effect, where the existence of long-range magnetic interactions leads to the softening at the ZB. $\frac{19}{}$ On the other hand, whether this approach is capable of explaining the observed spin wave broadening remains unclear. Third, large magnon-phonon interactions may give rise to the remarkable $\mathrm{ZB}$ softening along specific directions $\stackrel{12}{=}$ Fourth, the deviation of short wave length 
magnons from the canonical Heisenberg form might originate from the scattering of magnons by collective quantum orbital fluctuations, which could either be planar $\left(x^{2}-y^{2}\right)$-type orbitals associated with the A-type antiferromagnetic (AF) ordering ${ }^{20,21}$ or rodlike $\left(3 z^{2}-r^{2}\right)$ orbital correlations related to C-type AF ordering. ${ }^{22}$ Depending on the actual orbital shape, the coupling between charge and orbital-lattice will give rise to distinct doping dependence of the softening/broadening of the magnetic spectrum. Fifth, the randomness created by the substitution of the divalent ions for the trivalent ions in $A_{1-x} B_{x} \mathrm{MnO}_{3}$ might be responsible for the anomalous spin wave softening. ${ }^{23,24}$ Finally, the overlap between the magnon excitations and Stoner continuum in the metallic $A_{1-x} B_{x} \mathrm{MnO}_{3}$ would cause softening and broadening of the magnon branch near ZB. ${ }^{25,26}$ Although this singleband DE model with intermediate coupling can explain the softening/broadening in the low- $T_{c}$ compounds, remarkable similarities in systems with widely different $T_{c}$ 's indicate it is inadequate as the bandwidth of Stoner Continuum is directly related to the $T_{c}$ 's.

Given that there are so many possible models to explain the ZB magnon softening, it is imperative to carry out systematic spin wave measurements and compare the results with predictions of various models. In a recent Letter $^{17}$, we made such comparison for spin waves along the $[\xi, 0,0]$ direction and found that none of prevailing models can account for the data. In this article, we expand our previous work and describe a systematic investigation of spin wave excitations of the CMR manganites $\mathrm{La}_{0.75} \mathrm{Ca}_{0.25} \mathrm{MnO}_{3}$ (LCMO25) and $\mathrm{La}_{0.70} \mathrm{Ca}_{0.30} \mathrm{MnO}_{3}$ (LCMO30). Using reactor based and time-of-flight inelastic neutron scattering (INS) techniques, we were able to map out the low temperature ferromagnetic spin wave excitations of LCMO25 and LCMO30 throughout the Brillouin zone in all symmetry directions. In the long wavelength limit, spin wave stiffness of LCMO25 and LCMO30 are $147 \pm 3$ and $169 \pm 2$ meV $\AA^{2}$ respectively, consistent with previous results 16,17 . At large wavevectors, we find that the dispersion relations of LCMO30 are considerable more renormalized (softened) in all major symmetry directions compared to those of LCMO25. The softening is well described by the introduction of the $4^{\text {th }} \mathrm{NN}$ ferromagnetic exchange coupling $J_{4}$ [Fig. 1(b)], the ratio of $J_{4} / J_{1}$ is about $19.5 \%$ in LCMO30 and $6.5 \%$ in LCMO25. In section II, we describe experimental details. Section III gives the data analysis and comparison with previous work. The conclusions are summarized in Section IV.

\section{EXPERIMENTAL DETAILS}

We grew single crystals of LCMO25 and LCMO30 using the traveling solvent floating zone technique. The Curie temperatures of LCMO25 $\left(T_{c}=190 \pm 1 \mathrm{~K}\right)$ and LCMO30 $\left(T_{c}=238 \pm 1 \mathrm{~K}\right)$ are determined from the elastic neutron diffraction on the (100) and (110) magnetic
Bragg peaks. 27 LCMO25 has a nominal hole doping level of $x=0.25$, just above the metal-insulator transition concentration $(x=0.22)$. LCMO30 has a doping level close to optimal doping with highest $T_{c}$. Our INS experiments were performed on the HET chopper spectrometer at the ISIS spallation neutron source, RutherfordAppleton Laboratory, and on the HB1/HB3 triple-axis spectrometers at the High-Flux-Isotope Reactor (HFIR), Oak Ridge National Laboratory. The momentum transfer wavevectors $q=\left(q_{x}, q_{y}, q_{z}\right)$ are in units of $\stackrel{\circ}{A}^{-1}$ at positions $(H, K, L)=\left(q_{x} a / 2 \pi, q_{y} b / 2 \pi, q_{z} c / 2 \pi\right)$ in reciprocal lattice units (rlu), where $a \approx b \approx c \approx 3.87 \AA$ and $3.86 \AA$ are the lattice parameters of the pseudocubic unit cells of LCMO25 and LCMO30, respectively [Fig. 1(a)]. The samples were aligned in the $(H, H, L)$ zone in both the ISIS and HFIR experiments. For the ISIS measurements, we use the HET direct-geometry chopper spectrometer which has the ${ }^{3} \mathrm{He}$ filled detector tubes covering the scattering angles from 9-29 ${ }^{\circ}$ (PSD detectors covers from $\left.2.5-7.9^{\circ}\right)$. For the HFIR experiment, we use pyrolytic graphite as monochromator, analyzer, and filters, and the final neutron energy was fixed at $E_{f}=13.5$ or $14.7 \mathrm{meV}$.
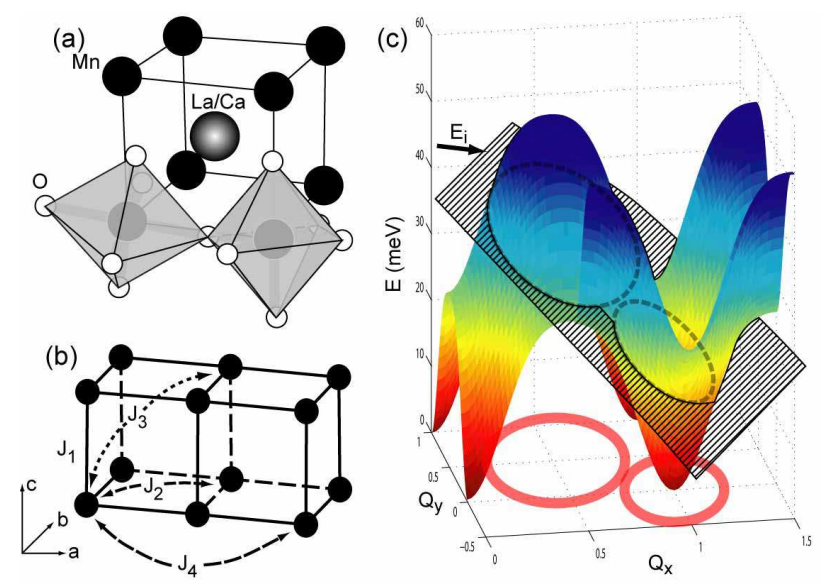

FIG. 1: (Color online) (a) Crystal structure of $\mathrm{La}_{1-\mathrm{x}} \mathrm{Ca}_{\mathrm{x}} \mathrm{MnO}_{3}$ with distorted oxygen octahedra surrounding $\mathrm{Mn}$ ions. (b) Magnetic exchange interactions up to $4^{\text {th }}$ order between adjacent $\mathrm{Mn}$ ions. (c) Illustration of time-of-flight experiment. The energy-wavevector $(E-q)$ region probed by the experiment intersects the spin wave dispersion surface, giving rise to spin wave rings projected on the scattering plane. For smaller energy transfer $E$, the ring centers at the zone center. For larger $E$, the ring centers at the ZB.

For the HET measurements, the magnetic scattering intensities of the raw data were normalized to a vanadium standard. The scattering function

$$
S(\mathbf{q}, \omega)=\frac{\left|k_{i}\right|}{\left|k_{f}\right|} \frac{d^{2} \sigma}{d \Omega d \omega},
$$

where $k_{i}$ and $k_{f}$ are the initial and final neutron wavevectors, respectively, the solid angle of scattering is $\Omega$, and 
the energy transfer is $\hbar \omega$. We used the program MSLICE ${ }^{28}$ to visualize the $\mathbf{q}-\omega$ data sets and to prepare the onedimensional cuts along the high-symmetry spin wave directions, needed for further analysis using the program TOBYFIT 29 .

\section{MODELING AND ANALYSIS}

The neutron scattering cross-section per formula unit (f.u.) for spin wave excitations is

$$
\frac{d^{2} \sigma}{d^{2} \Omega d \omega}=\left(\gamma r_{0}\right)^{2} \frac{\left|k_{f}\right|}{\left|k_{i}\right|}|F(\mathbf{q})|^{2} \frac{1}{\pi g^{2} \mu_{B}^{2}} \frac{1}{1-e^{-\beta \hbar \omega}} \chi^{\prime \prime}(\mathbf{q}, \omega) \text {, }
$$

where $\left(\gamma r_{0}\right)^{2}=0.2906$ barn, $|F(\mathbf{q})|^{2}$ is the magnetic form factor, $g$ is the Lande factor $(\approx 2),[n(\omega)+1]=$ $1 /\left[1-\exp \left(-\hbar \omega / k_{B} T\right)\right]$ is the detailed balance factor and $\mu_{B}$ is the Bohr magneton. $\chi^{\prime \prime}(\mathbf{q}, \omega)$ is the imaginary part of the generalized spin susceptibility which depends on the wavevector $q$ and energy transfer $\hbar \omega$. In the damped simple harmonic oscillator (DSHO) approximation, the normalized dynamical susceptibility $\chi^{\prime \prime}(\mathbf{q}, \omega)$ can be written as

$$
\chi^{\prime \prime}(\mathbf{q}, \omega)=\frac{4 \gamma \omega \omega_{0}}{\pi\left[\left(\omega^{2}-\omega_{0}^{2}\right)^{2}+4(\gamma \omega)^{2}\right]},
$$

where $\gamma$ characterizes the damping of the magnetic spins, $\omega_{0}$ is directly associated with the spin wave dispersion relation. In the light damping limit, the intrinsic peak height $A$ and width $\Gamma$ of the spin wave excitation profiles are determined by the damping $\gamma$ and become

$$
A \propto 1 /(2 \pi \gamma), \Gamma \propto 2 \gamma .
$$

The Hamiltonian for a Heisenberg ferromagnet is

$$
H=-\frac{1}{2} \sum_{i, k} J_{k} \mathbf{S}_{i} \cdot \mathbf{S}_{i+k}
$$

where $\mathbf{S}_{i}$ denotes the magnetic moment at site $i$, and $J_{k}$ indicates the magnetic exchange coupling between neighboring sites. In an early study, a $\mathrm{NN}$ coupling $J_{1}$ has been successfully employed to describe the entire spin wave dispersion relation $E(\mathbf{q})$ for high- $T_{c}$ manganites ${ }^{7}$ Subsequent measurements have shown the presence of $\mathrm{ZB}$ spin wave softening for all other manganites with dispersions being reproduced well by a Heisenberg Hamiltonian with higher order interactions $10,17,22$ Recently, we found that the introduction of the $4^{\text {th }} \mathrm{NN}$ interactions $J_{4}$ gives satisfactory description of ZB softening of the spin wave dispersions for a wide range of doped manganites along the $[\xi, 0,0]$ direction. ${ }^{17}$ We show here that such model also gives reasonable description of spin waves in all other symmetry directions.

\section{A. Results on $\mathrm{La}_{0.75} \mathrm{Ca}_{0.25} \mathrm{MnO}_{3}$}

LCMO25 undergoes a ferromagnetic phase transition and becomes metallic below $T_{c}=190 \mathrm{~K}{ }^{27}$ To determine the dispersion of LCMO25 at large momentum transfers, we measured its ferromagnetic spin waves with incident beam neutron energies $\left(E_{i}\right)$ of $32,50,75,100,125,150$, 175 , and $185 \mathrm{meV}$ at $T=8.5 \mathrm{~K}\left(0.045 T_{c}\right)$ on HET. The sample was oriented such that either the $[1,1,0]$ or $[0,0,1]$ axis of the crystal is along the incident beam directions. For the neutron beam along the $[1,1,0]$ axis of the crystal, we could get the dispersion relations along the $[\xi, 0,0]$ or $[\xi, \xi, 0]$ directions. For the neutron beam along the $[0,0,1]$ axis, the dispersion along the $[\xi, \xi, \xi]$ direction can be obtained.

Fig. 2 summarizes the spin wave excitations for incident neutron energies of 50, 75, and $100 \mathrm{meV}$. Panels (ac) show the two-dimensional color-coded contour plots of excitations in reciprocal space. Two rings of scattering are observed in these panels. The first and strongest of these two is centered at the $(1,0,0)$ and corresponds to the intersection of the $E-q$ region probed by the experiment, and the spin wave dispersion surface near the zone center. The second of these rings, centered at $(1.5,0.5,0)$, corresponds to such intersections near the ZB because of a larger energy transfer $E$ [Fig. 1(c)]. We cut the $E-q$ data along the $[\xi, 0,0]$ direction, as shown in panels $(\mathrm{d}-$ f). The cut clearly shows two distinct peaks located at 11.7 and $16.3 \mathrm{meV}$ for $E_{i}=50 \mathrm{meV}$ [Fig. 2(d)]. These peaks gradually disperse outward with increasing incident beam energy [Figs. 2(e),(f)]. As a function of increasing energy and approaching the ZB wavevector, the spin waves become broader in width and weaker in intensity [Fig. 2(f)]. These results are consistent with our earlier measurements. 17

To obtain spin wave excitations along the $[\xi, \xi, 0]$ direction, we change the orthogonal viewing axes of the two-dimensional spin wave spectra and cut images along the $[\xi, \xi, 0]$ direction. Fig. 3] summarizes the outcome of these cuts, it is clear that the spin wave peaks become broader and weaker near the ZB [Fig. [3(c),(f)].

We systematically cut the data along all high symmetry directions for various incident beam energies. To obtain reliable exchange coupling constant $J_{i}$ 's and determine their directional dependence, we analyze data along one symmetry direction at a time with as many cuts as possible. The data are fit simultaneously using the dynamic susceptibility $\chi^{\prime \prime}(\omega, \mathbf{q})$ described in Eqn. 3 with spin wave dispersion relation as

$$
\hbar \omega_{0}(\mathbf{q})=\Delta+2 S[J(0)-J(\mathbf{q})],
$$

where $\Delta$ is the anisotropic gap and

$$
J(\mathbf{q})=\sum_{i} J_{k} e^{i \mathbf{q} \cdot\left(\mathbf{R}_{i}-\mathbf{R}_{j}\right)} .
$$

Using the TOBYFIT non-linear least-square analysis program, we fit all the cuts by adjusting the peak amplitude $A$, damping term $\Gamma$ and magnetic exchange coupling constant $J_{i}$ 's. The time-of-flight measurements do not provide information at small momentum transfers, but the data obtained using triple-axis spectroscopy 

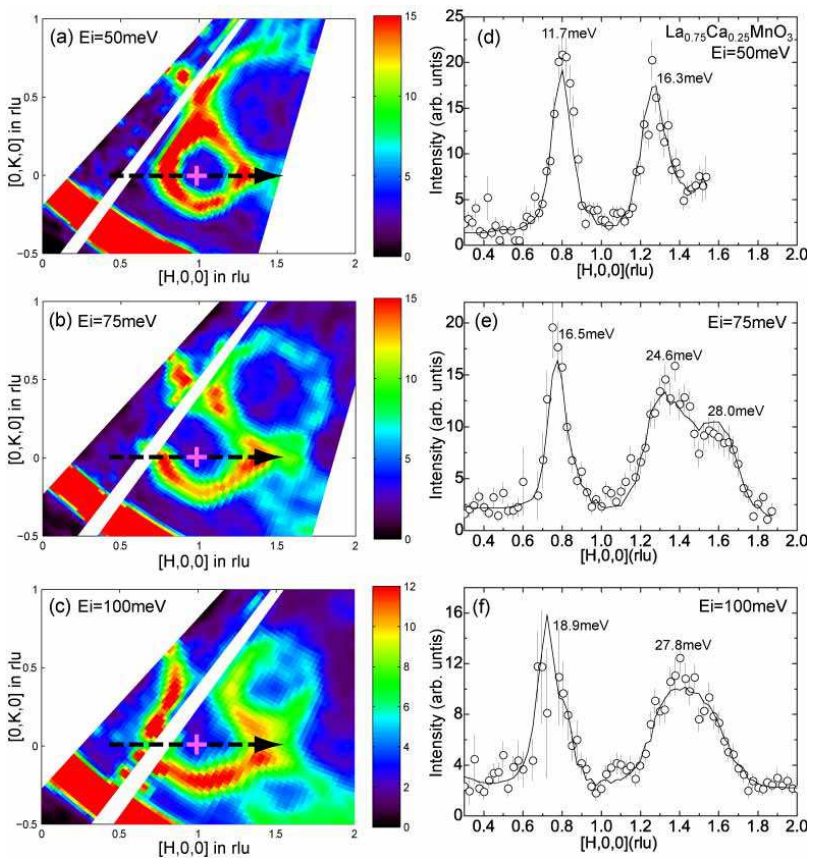

FIG. 2: (Color online) Spin wave excitations of LCMO25 in the $[\xi, 0,0]$ direction. Panels (a-c) illustrate the 2D contour plots in the reciprocal space with incident energy of 50, 75, and $100 \mathrm{meV}$. The cross symbols indicate the FM zone center of $(1,0,0)$. Panels (d-f) depict the corresponding scan profiles along the $[\xi, 0,0]$ direction shown by the arrows in the left panels. The typical cut width is 0.1 rlu. Solid lines are least square fits using the spin wave model described in the text. The energies associated with excitation peaks are labeled.

showed negligible anisotropic spin gap near the zone center $10,11,12,13,14$ We therefore fixed $\Delta=0$ in Eqn. 6 during the analysis. Following the results of our previous paper, we force $J_{2}$ and $J_{3}$ to be zero during the course of analysis, allowing only $J_{1}$ and $J_{4}$ to vary. 17 Table I shows the fitting results in LCMO25 along three major symmetry directions of $[\xi, 0,0],[\xi, \xi, 0]$ and $[\xi, \xi, \xi]$. We note that the value of exchange constants varies in a narrow range for fitted results along different directions. For example, the NN exchange coupling $J_{1}$ changes from 7.6 to $8.4 \mathrm{meV}$ and $J_{4}$ varies from 0.26 to $0.58 \mathrm{meV}$. Using $D=\left.\nabla_{\mathbf{q}}^{2} \omega_{0}(\mathbf{q})\right|_{\mathbf{q}=0}$, where $\omega_{0}(\mathbf{q})$ is the dispersion relation, we can calculate the spin wave stiffness constant $D$ and found that $D \approx 145 \mathrm{meV} \AA^{2}$, consistent with the value obtained from low- $q$ inelastic scattering measurement $\frac{17}{17}$ Since the fitting results along the three high symmetry directions are consistent, we included excitation data along all directions in the товYFIт. As shown in Table 【, the global analysis with as many as 38 data sets along all directions gives $J_{1}=7.83 \pm 0.06$ and $J_{4}=0.51 \pm 0.03 \mathrm{meV}$, which yields the ratio of $J_{4} / J_{1}=0.065 \pm 0.004$ and stiffness constant $D=147.2 \pm 2.6 \mathrm{meV} \AA^{2}$.

TOBYFit gives only the fitting parameters $J_{i}$ 's, $A$ and $\Gamma$ for the spin wave dispersion relations. To actually construct a plot of $E$ versus $q$ of $\mathrm{LCMO} 25$, we derive the
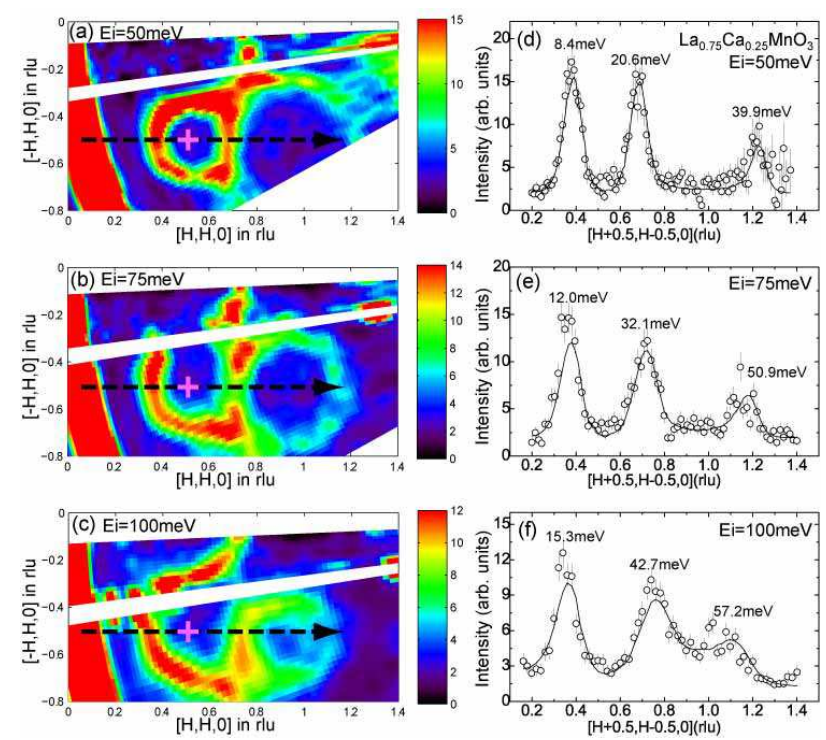

FIG. 3: (Color online) Spin wave excitations of LCMO25 in the $[\xi, \xi, 0]$ direction with incident energies of 50,75 , and $100 \mathrm{meV}$. The viewing axis of panels (a-c) are rotated $45^{\circ}$ with respect to those in Fig. 2 such that the scan profiles along $[\xi, \xi, 0]$ can be obtained. The cross symbols indicate the FM zone center of $(1,0,0)$. The dashed lines depict the cutting direction.

energy at an individual wavevector $E(q)$ by analyzing each single one-dimensional cuts as shown in Figs. 2(df) $\stackrel{30}{=}$ As shown in Fig. 4 the experimental data collapse nicely onto the solid line generated using Eqns. 6] and 7 with inclusion of the $\mathrm{NN}$ interaction $J_{1}$ and $4^{\text {th }} \mathrm{NN}$ interaction $J_{4}$. Finally, we show that a fit to a purely NN exchange coupling $J_{1}$ is not adequate. The dispersion relations based only on $J_{1}$ are plotted as the dashed lines, although these curves describe reasonable well the low- $q$ data, they clearly deviate the data points at the ZB with higher energies. For example, the actual ZB energies along $[\xi, 0,0]$ and $[\xi, \xi, 0]$ directions are 31.0 and $62.5 \mathrm{meV}$, respectively. They are lowered by about 4 and $8 \mathrm{meV}$ from the prediction of the NN Heisenberg Hamiltonian. It is noted that while the description using only the NN exchange coupling is not sufficient to characterize the entire dispersion relations in LCMO25, this exchange constant $J_{1}$ is similar to the value established in the INS study of $\mathrm{La}_{0.7} \mathrm{~Pb}_{0.3} \mathrm{MnO}_{3} ?^{\underline{7}}$

\section{B. Results on $\mathrm{La}_{0.7} \mathrm{Ca}_{0.3} \mathrm{MnO}_{3}$}

To determine the evolution of spin wave excitations of ferromagnetic CMR compounds, we also measured LCMO30 using HET. For these measurements, we used incident beam energies of 50,75, 100, 125, 150 and 185 meV. Fig. [5 and Fig. [6 show raw data for LCMO30 with the same incident energies as those of LCMO25 in Figures 2 and 3. It is clear that LCMO30 has a 


\begin{tabular}{|c|c|c|c|c|c|c|}
\hline Direction & $J_{1}(\mathrm{meV})$ & $J_{4}(\mathrm{meV})$ & $J_{4} / J_{1}$ & $D\left(\mathrm{meV}^{2}\right)$ & No. of data sets & $\chi^{2}$ \\
\hline$[\xi, 0,0]$ & $7.56(7)$ & $0.58(4)$ & $0.076(6)$ & $147.1 \pm 3.5$ & 11 & 1.45 \\
\hline$[\xi, \xi, 0]$ & $7.99(9)$ & $0.44(4)$ & $0.055(6)$ & $145.6 \pm 3.9$ & 13 & 1.81 \\
\hline$[\xi, \xi, \xi]$ & $8.39(14)$ & $0.26(8)$ & $0.032(10)$ & $140.7 \pm 6.6$ & 14 & 2.09 \\
\hline$[\xi, 0,0]+[\xi, \xi, 0]+[\xi, \xi, \xi]$ & $7.83(6)$ & $0.51(3)$ & $0.065(4)$ & $147.2 \pm 2.6$ & 38 & 1.90 \\
\hline$[\xi, 0,0]+[\xi, \xi, 0]+[\xi, \xi, \xi]$ & $8.75(3)$ & 0 (Fixed) & 0 & $130.3 \pm 0.5$ & 38 & 2.32 \\
\hline
\end{tabular}

TABLE I: Fitting parameters of the exchange coupling constant $J_{1}, J_{4}$, the ratio of $J_{4} / J_{1}$ and spin wave stiffness constant $D$ in LCMO25. Each data set is one dimensional scan profile as shown in Fig. 2(d-f) and consists of one or more magnetic excitation peaks. The results with only the NN interaction $J_{1}$ are listed in the last row for comparison.

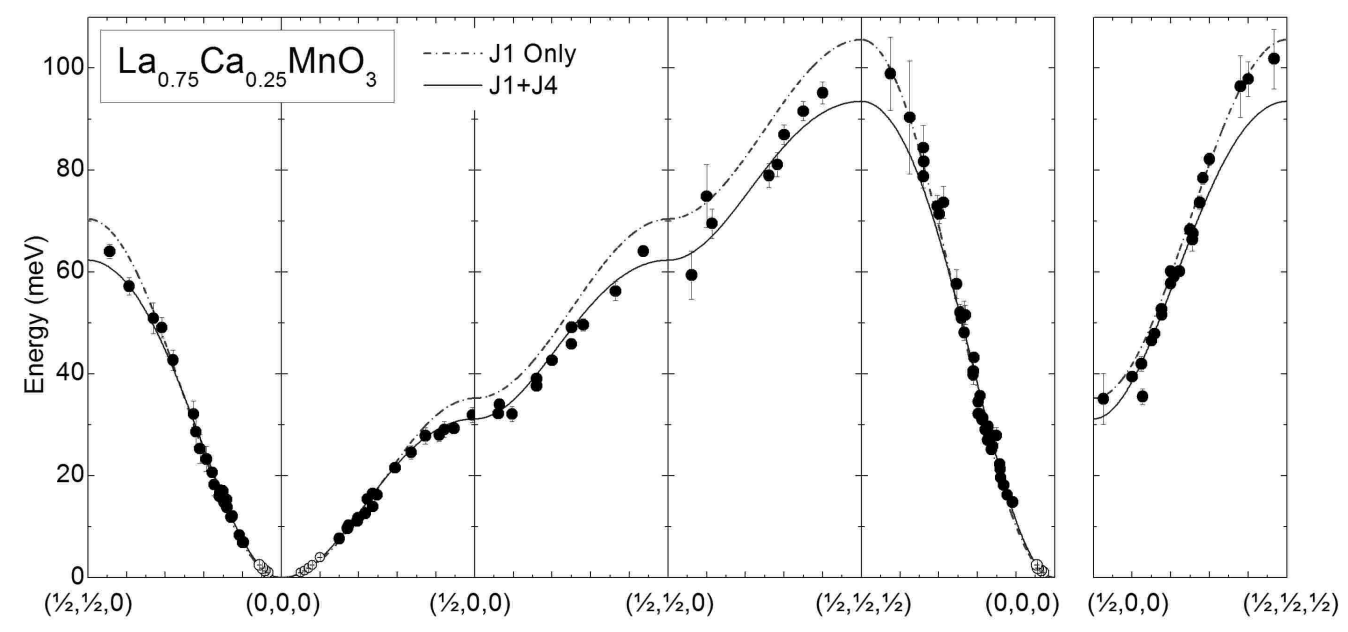

FIG. 4: (Color online) The spin wave dispersion curves for LCMO25. The solid points are data collected at ISIS and the open points at low energies are collected at HFIR. The dash (red) curves are the fits using only the NN interaction $J_{1}$. The solid (blue) curves are the fits combining the NN interaction $J_{1}$ and the $4^{\text {th }} \mathrm{NN}$ interaction $J_{4}$ (see text).

softened dispersion near the ZB. As shown in Fig. 5 (f), the peak height at $q=[1.4,0,0]$ is only half of that at $q=[0.7,0,0]$, compared with $80 \%$ for that in LCMO25 [Fig. 2(f)]. From Eqn. 4, the decrease in peak height at a similar wavevector indicates a larger damping term $\gamma$. In addition, the spin wave ring away from $(1.5,0.5,0)$ [Fig. 6(c),(f)] collapses much faster than in LCMO25, as the incident beam energy increases to $100 \mathrm{meV}$. Table II lists the quantitative fitting parameters using TOBYFIT. Much reduced $J_{1}$ and considerable enhanced $J_{4}$ are observed along different high symmetry directions for LCMO30. Similar to LCMO25, the softening near the boundary shows little symmetry directional dependence. For the global fit, we obtained $J_{1}=6.36 \pm 0.03$ and $J_{4}=1.24 \pm 0.02 \mathrm{meV}$. The ratio of $J_{4} / J_{1}$ reaches $20 \%$, a value much larger than that in LCMO25. The stiffness constant is calculated to be $D=169 \pm 2 \mathrm{meV} \AA^{2}$, agrees well with the early triple-axis scattering result $\underline{16,31}$

The dispersion curves covering the entire Brillouin Zone of LCMO30 are shown in Fig. 7 with the solid lines representing the fits using $J_{1}$ and $J_{4}$, while the dashed lines representing the fits using only $J_{1}$. Inspection of Fig. 7 reveals that, as in LCMO25, the inclusion of the $J_{4}$ gives a better fit to the data. The normalized chisquared, $\chi^{2}$, including $J_{4}$ is a factor of 2 less than that without it. The renormalization of spin wave dispersions at large wavevectors is better seen in the $[\xi, 0,0]$ and $[\xi, \xi, 0]$ directions, where the boundary energies are lowered to 25 and $52 \mathrm{meV}$, respectively. They are nearly 9 and $15 \mathrm{meV}$ lower than the expectation from a simple NN Heisenberg Hamiltonian. We note that fits at larger energies $(E>70 \mathrm{meV})$ are not satisfactory, possibly because uncertainties associated with the determination of a already damped magnetic excitation at high energies.

Having determined the dispersion relations for LCMO25 and LCMO30 using the model described in Eqn. 6 with inclusion of $J_{1}$ and $J_{4}$, we now consider the other important aspect of the spin dynamics, the intrinsic width $\Gamma(\mathbf{q})$. This linewidth $\Gamma(\mathbf{q})$ is calculated from Eqn. 4, where $\gamma$ is obtained from the best fit to Eqn. 3. It is directly associated with the relevant damping mechanisms and reflects how the quantized magnons interact with other scattering processes. The wavevector dependence of $\Gamma(\mathbf{q})$ along different directions is shown in Fig. 8 , Note that there is marked difference between LCMO25 and LCMO30; the widths of the latter are always larger, indicating more damped excitations in LCMO30. In addition, the momentum evolution of $\Gamma(\mathbf{q})$ does not show any anomaly across the Brillouin zone, appear to be isotopic and increase drastically near the ZB. For example, 


\begin{tabular}{|c|c|c|c|c|c|c|}
\hline Direction & $J_{1}(\mathrm{meV})$ & $J_{4}(\mathrm{meV})$ & $J_{4} / J_{1}$ & $D\left(\mathrm{meV}^{2}\right)$ & No. of data sets & $\chi^{2}$ \\
\hline$[\xi, 0,0]$ & $6.01(5)$ & $1.29(4)$ & $0.215(8)$ & $166.6 \pm 2.8$ & 13 & 1.71 \\
\hline$[\xi, \xi, 0]$ & $6.52(5)$ & $1.18(4)$ & $0.181(6)$ & $167.4 \pm 2.5$ & 22 & 1.91 \\
\hline$[\xi, \xi, \xi]$ & $6.56(9)$ & $1.18(6)$ & $0.179(11)$ & $167.8 \pm 4.8$ & 15 & 1.54 \\
\hline$[\xi, 0,0]+[\xi, \xi, 0]+[\xi, \xi, \xi]$ & $6.36(3)$ & $1.24(2)$ & $0.195(4)$ & $168.6 \pm 1.7$ & 50 & 1.74 \\
\hline$[\xi, 0,0]+[\xi, \xi, 0]+[\xi, \xi, \xi]$ & $8.43(3)$ & 0 (Fixed) & 0 & $125.7 \pm 0.5$ & 50 & 3.25 \\
\hline
\end{tabular}

TABLE II: Fitting parameters of exchange coupling constant $J_{1}, J_{4}$, the ratio of $J_{4} / J_{1}$ and spin wave stiffness constant $D$ in LCMO30. See Table【 for additional information.
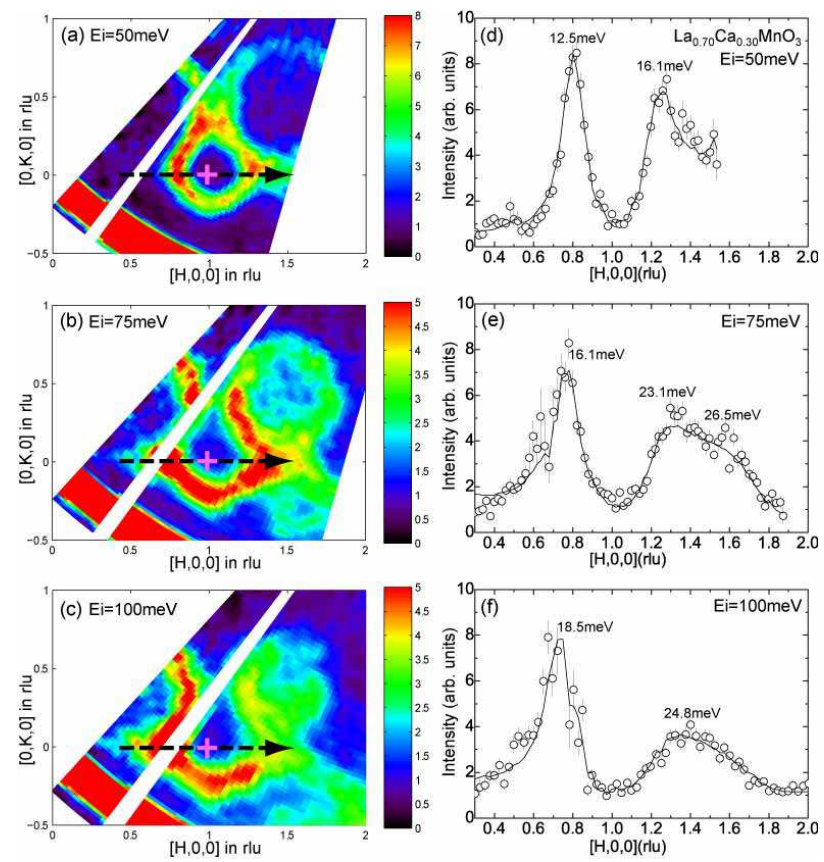

FIG. 5: (Color online) Spin wave excitations of LCMO30 in the $[\mathrm{H}, 0,0]$ direction. Panels (a-c) illustrate $2 \mathrm{D}$ contour plots with incident energies of 50, 75, and $100 \mathrm{meV}$. Solid lines are least square fits. See Fig. 2 for additional information.

$\Gamma(\mathbf{q})$ reaches around $20 \mathrm{meV}$ for $E$ greater than $60 \mathrm{meV}$ along the $[\xi, \xi, \xi]$ direction. This is unexpected from a classic, cubic Heisenberg ferromagnet, where the magnetic excitations near the $\mathrm{ZB}$ are well resolved ${ }^{32}$ The intrinsic widths in both LCMO25 and LCMO30 are much broader, indicating that one or more decay mechanisms play an important role in these CMR compounds.

We are now in a position to compare our data to various possible mechanisms of the ZB softening. First, we want to comment on the role of disorder or randomness which is naturally present in these compounds. Motome and Furukawa 23 have pointed out that disorder in CMR materials will cause anomalous broadening and/or anticrossing in the spin excitation spectra. In this scenario, the one-electron bandwidth is proportional to the average ionic-size at La/Ca site $\left(\bar{r}=\sum_{i} x_{i} r\right.$, where $x_{i}$ is the fractional occupancies of $A$-site species, $r_{i}$ is the individual radius). In a previous paper, we have characterized the softening of dispersion relation in a series of doped man-
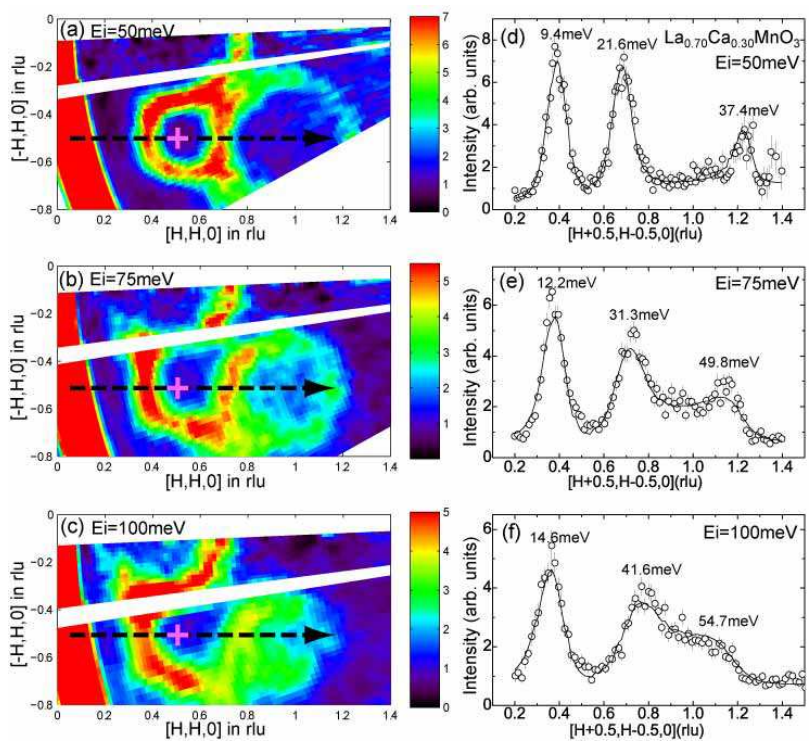

FIG. 6: (Color online) Spin wave excitations of LCMO30 in the $[\mathrm{H}, \mathrm{H}, \mathrm{O}]$ direction with incident energies of 50,75 , and $100 \mathrm{meV}$. See Fig. 2 for additional information.

ganites near $x=0.30$ along $[\xi, 0,0]$ direction $\stackrel{17}{=}$ We found the renormalization near ZB has little dependence on the average ionic size at $A$-site. For LCMO25 and LCMO30, $\bar{r}$ become 1.207 and $1.205 \AA$, respectively, showing very little variation of oxygen octahedron distortion surrounding the Mn-ions. On the other hand, the mismatch between La and $\mathrm{Ca}$ ions will cause a quenched disorder in the system, which can be qualitatively characterized by $\sigma^{2}=\sum_{i}\left(x_{i} r_{i}^{2}-\bar{r}^{2}\right)^{33.34}$ Base on this, $\sigma^{2}$ is $2.43 \times 10^{-4}$ for LCMO25 and $2.72 \times 10^{-4}$ for LCMO30. Clearly, the differences in quenched disorder between LCMO25 and LCMO30 are rather small and cannot account for the dramatic change of spin wave spectra near the ZB. We therefore conclude that the disorder effect does not play an important role in this doping range of LCMO.

Second, the effect of magnon-phonon coupling may be the microscopic origin of the observed magnon broadening and damping. Dai and co-workers suggest that the interaction between optical phonon and spin wave branches may lead to the broadening of spin wave spectrum in a number of doped manganites near $x=0.30 \underline{12}$ In this picture, a dispersionless optical phonon branch 


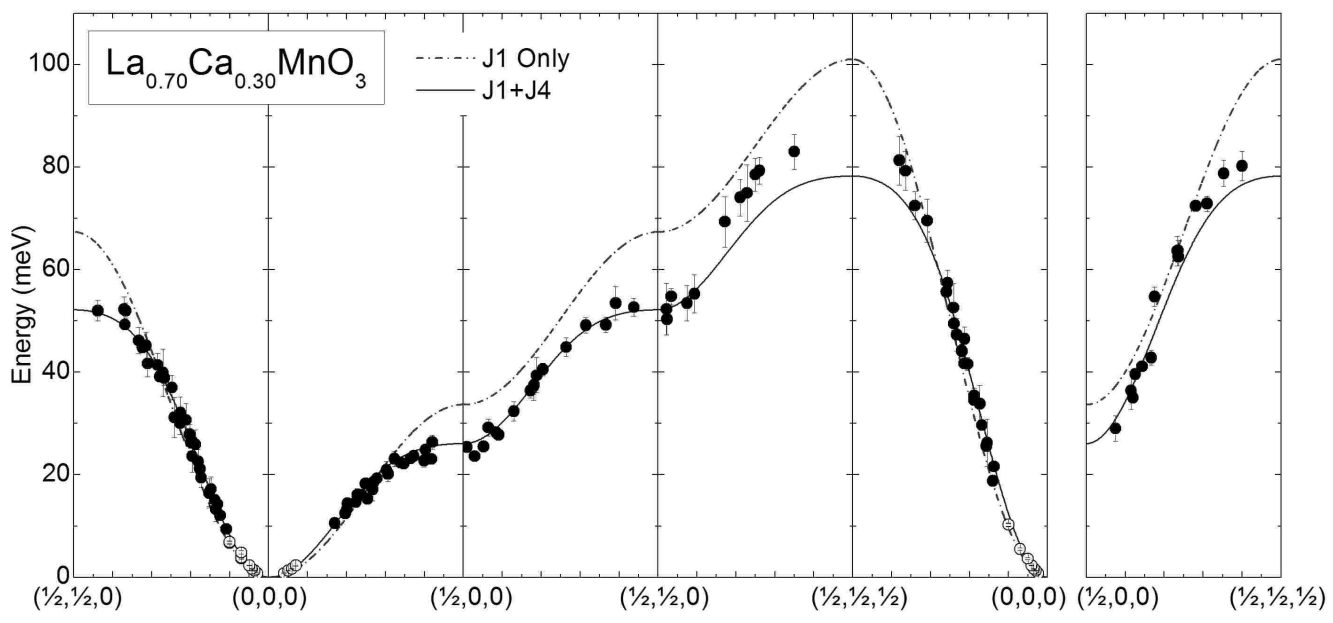

FIG. 7: (Color online) The spin wave dispersion curves for LCMO30. The dash (red) curves are the fits using only NN interaction $J_{1}$. The solid (blue) curves are the fits using $J_{1}$ plus the $4^{\text {th }}$ NN interaction $J_{4}$.

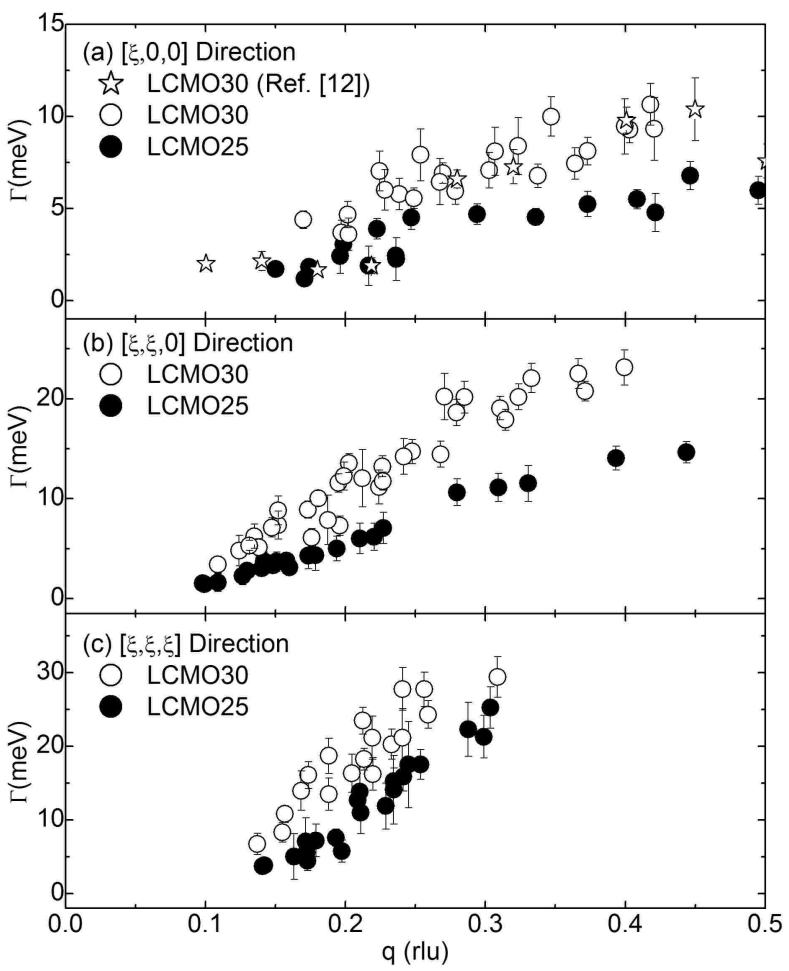

FIG. 8: The wavevector dependence of intrinsic excitation widths $\Gamma(\mathbf{q})$ along three high symmetry directions in LCMO25 and LCMO30. The $q$-dependence of $\Gamma(\mathbf{q})$ along $[\xi, 0,0]$ for LCMO30 obtained from triple-axis measurement (Ref. 12]) is displayed for comparison.

with energy around $20 \mathrm{meV}$ goes across the whole Brillouin zone and interacts with the magnon branch, causing softening and a substantial increase of the magnetic excitation linewidths. The measurements of Dai et al. were carried out with unpolarized neutrons, and it was difficult to separate the magnetic scattering from the purely lattice excitations. Recent measurements by FernandezBaca et al $\frac{35}{5}$ using polarized neutron scattering techniques confirmed that the ZB spin waves of LCMO30 in the $[\xi, 0,0]$ and $[\xi, \xi, 0]$ directions are considerable broad and have energies lower than those expected from the approximation to the NN Heisenberg Hamiltonian, although the softening in $[\xi, \xi, 0]$ is less as severe than originally reported. 36 While the magnon-phonon interaction mechanism seems to explain the broadening of the spin waves, it is not clear if it would fully account for the magnitude of the observed softening in LCMO30 at the zone boundary. Furthermore, this mechanism may not be relevant to the general case of the manganites as the observed spin waves in the $\mathrm{Sm}_{0.55} \mathrm{Sr}_{0.45} \mathrm{MnO}_{3}$ are softened to around $15 \mathrm{meV}$ (which is below the $20 \mathrm{meV}$ optical phonon) at the $\mathrm{ZB}$ along $[\xi, 0,0]$ direction, with no evidence of broadening ${ }^{22}$

Finally, the distinct feature of ZB softening might be a consequence of the $e_{g}$-band filling in the halfmetallic region. $\frac{19}{}$ Solovyev and Terakura suggested that the canonical double exchange is no longer appropriate as soon as holes are doped into the system. Longer range FM interactions lead to the softening at the zone boundary and contribute to the increase of the stiffness constant $D \stackrel{19}{\underline{19}}$ However, the details of the realistic electronic structure are important and may significantly modify the analysis, particularly if the effects of $t_{2 g}$ electrons are taken into account. For example, the $x$ dependence of magnetic interactions is substantial modified by the change of DE interaction contributed by $t_{2 g}$ electrons and $D$ might even decrease with $x$. Such complex scenario prevents us to make meaningful comparison with the $D$ 's yield experimentally. It is also unclear whether this model can explain the commonly observed damping/broadening of magnetic excitation near the ZB. We would like to point out that, in the tight-binding approximation used by these authors, only the exchange couplings along the Mn-O-Mn chain $\left(J_{1}, J_{4}, J_{8}\right.$, and $J_{15}$ as defined in Ref. 19) 
would bring appreciable contributions to the spin wave dynamics. Our work, and that of Endoh, 22 show that $J_{1}$ and $J_{4}$ are the only exchange constants contributing to the ZB softening. There is close correlation between $J_{i}$ 's and the orbital polarized states $;^{22} J_{2}$ would be enhanced by $\left(x^{2}-y^{2}\right)$-type orbitals and $J_{4}$ is enhanced of $\left(3 z^{2}-r^{2}\right)$ type orbitals. It is surprising that a large increase of $J_{4} / J_{1}$ occurs despite of the small change in nominal hole doping. This might indicate a drastic modification of (preformed) orbital correlations which favor the overlap between neighboring Mn ions. 37 We hope that the results presented here will help to stimulate further experimental and theoretical investigations leading to a complete understanding of the magnetic dynamics in those CMR materials.

\section{CONCLUSIONS}

In summary, we have performed a systematic study on the spin wave dynamics in the CMR manganites LCMO25 and LCMO30 using inelastic neutron scattering techniques. We find that both systems display considerable spin wave renormalization along all high symmetry directions. Consistent with early measurements, which entirely focus on the $[1,0,0]$ direction, we find that the dispersion relations can be phenomenologically analyzed using the NN interaction $J_{1}$ and the $4^{\text {th }} \mathrm{NN}$ interaction $J_{4}$. The introduction of $J_{4}$ lowers $J_{1}$ and therefore lowers the ZB energy. As a result of this the systems with a larger $J_{4} / J_{1}$ ratio exhibit a larger SW softening at the ZB. The possible mechanisms responsible for such boundary softening are also discussed.

\section{ACKNOWLEDGMENTS}

We thank Thomas Enck for preliminary data analysis. The authors are grateful for the valuable discussion with Dr. M. E. Hagen. This work was supported by U.S. NSF DMR-0453804. ORNL is supported by U.S. DOE under Contract No. DE-AC05-00OR22725 with UT/Battelle LLC.
* Electronic address: yef1@ornl.gov

1 M. B. Salamon and M. Jaime, Rev. Mod. Phys. 73, 583 (2001).

2 Yoshinori Tokura, Colossal Magnetoresistive Oxides (Gordon and Breach Science Publishers, 2000).

3 E. Dagotto, T. Hotta and A. Moreo, Phys. Reports 344, 1 (2002).

4 C. Zener: Phys. Rev. 82, 403 (1951).

5 N. Furukawa, J. Phys. Soc. Jpn. 65, 1174 (1996).

6 M. C. Martin, G. Shirane, Y. Endoh, K. Hirota, Y. Moritomo, and Y. Tokura, Phys. Rev. B 53, 14285 (1996).

7 T. G. Perring, G. Aeppli, S. M. Hayden, S. A. Carter, J. P. Remeika, and S.-W Cheong, Phys. Rev. Lett. 77, 711 (1996).

8 Y. Endoh and K. Hirota, J. Phys. Soc. Jpn, 66, 2264 (1997).

9 Y. Motome and N. Furukawa, Phys. Rev. B 68, 144432 (2003).

10 H. Y. Hwang, P. Dai, S.-W. Cheong, G. Aeppli, D. A. Tennant, and H. A. Mook, Phys. Rev. Lett. 80, 1316 (1998).

11 L. Vasiliu-Doloc, J. W. Lynn, A. H. Moudden, A. M. de Leon-Guevara, and A. Revcolevschi, Phys. Rev. B 58, 14913 (1998).

12 P. Dai, H. Y. Hwang, J. Zhang, J. A. Fernandez-Baca, S.W. Cheong, C. Kloc, Y. Tomioka, and Y. Tokura, Phys. Rev. B 61, 9553 (2000).

13 T. Chatterji, L. P. Regnault, and W. Schmidt, Phys. Rev. B 66, 214408 (2002).

14 F. Moussa, M. Hennion, F. Wang, P. Kober, J. RodriguezCarvajal, P. Reutler, L. Pinsard, and A. Revcolevschi, Phys. Rev. B 67, 214430 (2003).

15 J. A. Fernandez-Baca, P. Dai, H. Y. Hwang, C. Kloc, and S.-W. Cheong, Phys. Rev. Lett. 80, 4012 (1998).

16 P. Dai, J. A. Fernandez-Baca, E. W. Plummer, Y. Tomioka, and Y. Tokura, Phys. Rev. B 64, 224429 (2001).
17 F. Ye, P. Dai, J. A. Fernandez-Baca, H. Sha, J. W. Lynn, H. Kawano-Furukawa, Y. Tomioka, Y. Tokura, and J. Zhang, Phys. Rev. Lett. 96, 047204 (2006).

18 D. I. Golosov, Phys. Rev. B 71, 014428 (2005).

19 I. V. Solovyev, and K. Terakura, Phys. Rev. Lett. 82, 2959 (1999).

20 G. Khaliullin and R. Kilian, Phys. Rev. B 61, 3494 (2000).

21 S. Krivenko, A. Yaresko, G. Khaliullin, and H. Fehske, J. Magn. Magn. Mater. 272-276 458 (2004).

${ }^{22}$ Y. Endoh, H. Hiraka, Y. Tomioka, Y. Tokura, N. Nagaosa, and T. Fujiwara, Phys. Rev. Lett. 94, 017206 (2005).

23 Y. Motome and N. Furukawa, J. Phys. Soc. Jpn. 71, 1419 (2002); and J. Phys. Soc. Jpn. 72, 472 (2003).

24 Y. Motome, and N. Furukawa, Phys. Rev. B 71, 014446 (2005).

25 X. Wang, Phys. Rev. B 57, 7427 (1998).

26 T. A. Kaplan, S. D. Mahanti, and Y.-S. Su, Phys. Rev. Lett. 86, 3634 (2001).

27 P. Dai, J. A. Fernandez-Baca, N. Wakabayashi, E. W. Plummer, Y. Tomioka, and Y. Tokura, Phys. Rev. Lett. 85, 2553 (2000).

28 R. Coldea, "MSLICE: A Data Analysis Programme for Time-of-Flight Neutron Spectrometers". (2004)

29 T. G. Perring, "TOBYFIT-Least-Squares Fitting to single crystal data on HET, MARI and MAPS". (2004)

30 In this approach, only $J_{1}$ is allowed to vary (the amplitude $A$ is fixed as its value has already been reliably determined from the global fitting, $J_{4}$ is fixed to be zero), the analysis would not carry the collective information of the whole dispersion relation but does provide the correct energy at a specific wavevector $q$.

31 J. W. Lynn, R. W. Erwin, J. A. Borchers, Q. Huang, A. Santoro, J.-L. Peng, and Z. Y. Li, Phys. Rev. Lett. 76, 4046 (1996).

${ }^{32}$ For instance, neutron scattering measurements on the 
prototype Heisenberg ferromagnet $\mathrm{EuO}$ show that the magnons broadening is small even at the zone boundary. H. A. Mook, J. A. Fernandez-Baca. (unpublished).

${ }^{33}$ L. M. Rodriguez-Martinez and J. P. Attfield, Phys. Rev. B 54, R15622 (1996).

34 R. D. Shannon, Acta Crystallogr. Sec. A 32, 751 (1976).

35 J. A. Fernandez-Baca, M. E. Hagen, Pengcheng Dai, F. Ye, J. Kulda, Y. Tomioka, and Y. Tokura, Physica B 385-386, 66 (2006).

36 The ZB magnon energy in the $[\xi, \xi, 0]$ direction of $52 \mathrm{meV}$ reported from our unpolarized neutron experiments is in agreement with the value $53 \pm 2 \mathrm{meV}$ measured by Fernandez-Baca et al. with polarized neutrons [35]. The reason of this agreement is attributed to the fact that using an incident energy of $100 \mathrm{meV}$ allowed us to measure the magnons in the $(1,0,0)$ Brillouin zone, where the magnetic scattering dominates the measured spectra.

37 The possibility of the ordering of complex orbitals in the ferromagnetic metallic manganites has been discussed by van den Brink and D. Khomskii. This type of orbital ordering would not introduce any lattice distortions, and would preserve the essentially cubic symmetry of these materials. J. van den Brink and D. Khomskii, Phys. Rev. B 63 , 140416(R) (2001). 